\title{
Recognition of Human Iris Using Accurate Iris Map
}

\author{
${ }^{1}$ T. Priyadharsini, M.Tech., ${ }^{2}$ K.Saminathan, M.Sc.,M.Phil.,M.Tech.,(Ph.D.,) \\ 1. PRIST UNIVERSITY Kumbakonam \\ ${ }^{2} \cdot$ Assistant Professor of CSE Dept PRIST UNIVERSITY Kumbakonam
}

\begin{abstract}
Personal identification based on Biometrics technology is a trend in future. Iris Recognition is regarded as a high accuracy verification technology when compared to traditional approaches. In real-time Iris Recognition, Iris Localization is very important step for Iris Recognition. So, the Iris Masks plays an important role in iris recognition. This Iris Mask indicates which part is occluded or contaminated by noisy image artifacts such as eyelashes, eyelids, eyeglasses frames, and specular reflections. If the Iris Mask is inaccurate, it will decrease its performance in Iris Recognition system. So, we use learning-based algorithms to estimate accurate Iris Masks from Iris Images, which propose to use Figueiredo and Jain's Gaussian Mixture Models (FJ-GMMs) to model the underlying probabilistic distributions of both valid and invalid regions on iris images. We also explored possible features and found that Gabor Filter Bank (GFB) provides the most discriminative information for our goal. Finally, we applied Simulated Annealing (SA) technique to optimize the parameters of GFB in order to achieve the best recognition rate.
\end{abstract}

Index Terms: Gaussian mixture models, iris mask, iris recognition, iris occlusion estimation, simulated annealing.

\section{Introduction}

Personal identification based on biometrics technology is a trend in the future. Iris recognition is regarded as a high accuracy verification technology, when compared t o traditional approaches. Iris segmentation refers to the process of extracting features that provide information of iris pattern. Normalization refers to preparing a segmented iris image for the encoding process. The next stage is the encoding technique, where the iris code is generated. Then, the iris code is stored in the database and it is useful for the verification process or matching.

In most cases, in the iris normalization stage, iris images are transformed from the Cartesian coordinate system to the polar coordinate system, as suggested by Daugman [1], [2], [3], [4], [5]. First, it normalizes the texture variation caused by changes in environmental illumination which lead to pupil dilation and contraction. The second advantage of this coordinate transformation is that it translates the rotational shift, i.e., eyeball inplane rotation due to head tilt. It also simplifies the problem in the matching stage. This effectively is handled by bit-shifting the whole iris code left/right by several bit columns to account for \pm 15 degrees of head rotation. Due to these two significant advantages, most iris recognition systems adopt this type of iris texture normalization in their implementation.

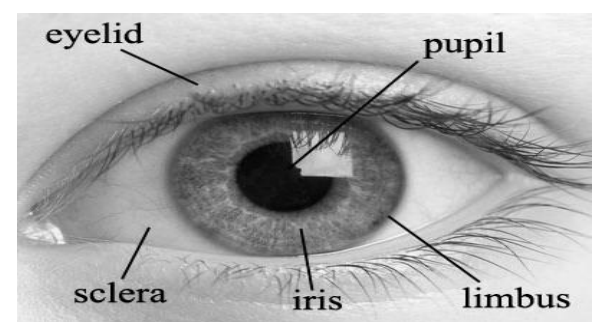

Fig. 1 Image of the eye

After transforming iris texture from the Cartesian coordinate to the polar coordinate, one has to create a mask for the iris map in the polar coordinate. The goal of this mask is to indicate which part in the iris map is truly iris texture and which part is noise. Traditionally, the main focus of iris recognition research addresses the power of the matching algorithm and the feature extraction. However, if the iris masks are inaccurate, the best feature extraction and recognition algorithms cannot compensate for such flaws. So this, accurate iris masks, combined with good features and effective recognition schemes, make the iris recognition systems more successful. 


\section{Previous Works -}

Daugman's work is one of the earliest in iris recognition [1]. Ma et al. proposed a full framework for an iris recognition system [6]. A similar assumption was proposed in the work of Tisse et al. [7]. Daugman, in a later work, proposed a more sophisticated algorithm for finding occluded regions in the iris images [5]. He proposed to locate the boundary of the eyelids at the segmentation stage. Kong and Zhang proposed a model for detecting eyelashes and specular reflections [9]. Zou et al. proposed a procedure for iris occlusion estimation [10]. In [11], Krichen et al. proposed a probabilistic approach for iris quality measure. In [12], Thornton proposed using a discriminative learning method based on Fisher Linear Discriminant Analysis (FLDA) to estimate iris masks in the polar domain. In [13] and [14], Sun et al. applied integrating Genetic Algorithms (GAs) on Gabor filter optimization for vehicle detection. In [15] and [16], different research groups were trying optimizing Gabor filters for the purpose of unsupervised texture recognition. Gabor filters are also used in biometrics applications. In [17] and [18], Gabor filters are used for feature extraction for the purpose of face recognition, and the authors were trying to optimize the parameters of Gabor filters in order to maximize the face recognition rate.

\section{Proposed Work -}

Previously, all works related to Iris Mask generation has some limitations. The parameter tuning of the Rule-Based approach is so important for the performance of the Iris Mask generation. And so the Rule-Based approach is very sensitive to the environmental setting for any specific database. Many Iris Masks generated are not accurate enough for Iris Recognition. So, to overcome the short-comings of these previous methods, we go for a Novel-Based approach. We propose a novel method for automatic iris mask generation and it has to be flexible for all possible sizes of Iris Images. This Novel method is called as Learning-Based approach, which has been introduced to achieve the goal of generating the accurate Iris Masks.

The aim of our paper is to correctly locate the Iris boundaries, i.e., both Pupil boundary and Limbus boundary form the Iris Image. It means finding the position of the centre and radius of the pupil and limbus to estimate the Iris Regions and to extract the Iris Masks during Iris Segmentation. This Iris Segmentation is done by Canny edge detector by applying the binary threshold image to separate the pupil and limbus from the image.

\section{Image Pre-Processing -}

The process of capturing the Iris Image is called as Enrolment. There are several devices for enrolment process such as Iris Sensor, DSLR camera and Mobile Iris camera. The Iris Image intensity depends on how the Iris captured whether using in-door device or outdoor device. In iris recognition system, iris region is the part between pupil and sclerotic, the aim of iris boundary localization is to locate the boundary of iris/pupil and the boundary of iris/sclerotic. Both inter boundary and outer boundary of iris are alike circles, so many iris localization methods are to locate iris boundaries using circle detector. Fast boundaries localization based on prior pupil centre position estimation can improve iris boundary localization real-time, the main idea of this algorithm is: firstly, pupil centre coarse localization, secondly, edge detection based on canny operation; thirdly, iris inter boundary localization in a small image block selected; fourthly, edge extraction based on local grey gradient extreme value; finally, outer boundary localization in image block selected based Hough transform.

\subsection{Pupil center coarse localization -}

In eye image, there are obvious lower grey levels in pupil regions than other parts as shown in Fig.2. Firstly, a binary threshold can be selected based on Histogram adopting p-tail method. Usually, iris image is captured in a distance, so pupil size is limited to a range in eye image, we can select threshold depend on the set rate of pupil pixels number to whole image pixels in histogram. Faculas can be seen in eye image as shown in Fig.2, these interferences must be removed, or they will affect iris boundary localization.

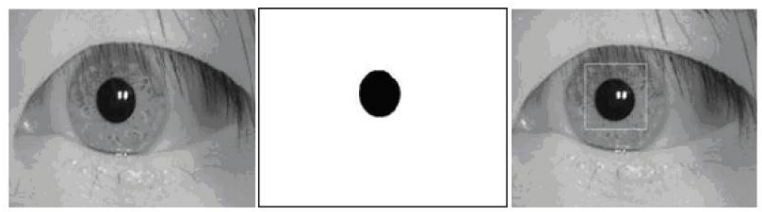

a) Original eye image b) Binarized result c) Pupil coarse estimation Fig . 2. Pupil centre coarse localization

According to high grey pixels caused by faculas in pupil parts, morphological operation can be used for filling with these holes and remove noise such as eyelashes by using close operation. So the original image of Fig.2 (a) can be transformed into the image of Fig.2 (b). After removing light-spots, pupil centre position will be estimated more accuracy, it is helpful to precision iris localization. 


\subsection{Iris outer boundary localization -}

In this paper, in order to realize iris boundary fast localization, a new method is proposed to extract iris edge points by using local gradient extreme value for each line of image, and then to achieve iris boundaries' parameters based on new voting approach. The upper eyelid and the lower eyelid often corrupt iris outer boundary, so we locate iris outer boundary by using part edge information. Because iris outer boundary has lower gradient than iris inter boundary (pupil boundary), it is very difficult to extract edges by comparing with a set threshold. If the threshold for edge extraction in iris gradient image is lower, then the more edge points extracted will be not helpful to improve real-time character of iris recognition system and the more edge points also may cause iris localization failure; if the threshold for edge extraction in iris gradient image is higher, then many edge points may be lost and it also can cause iris localization failure. So, we want to extract edge information by using local grey gradient extreme value, then to locate outer boundary based on Hough transform.

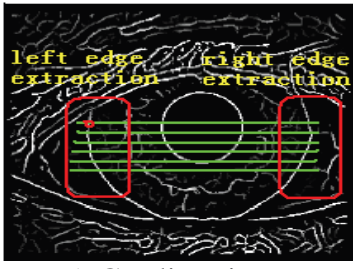

a) Gradient image

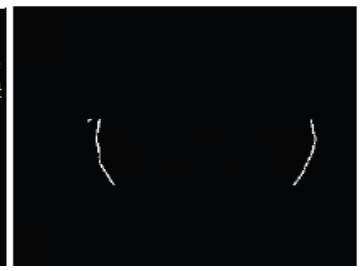

b) Binary image

Fig. 3 Binary edges extract principle based on line grey gradient extreme value

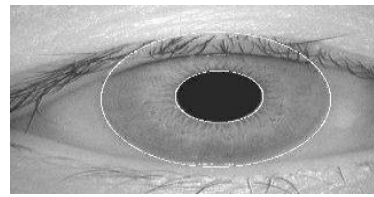

Fig. 4. Iris boundary localization results

Iris boundary localization algorithm is as follows:

Step 1. Pupil centre coarse localization;

Step 2. Select a small image block and extract edge information based on canny operator;

Step 3. Pupil boundary localization based on Hough transform;

Step 4. Select a small image block and extract edge information based on line's grey gradient extreme value;

Step 5. Iris outer boundary localization based on Hough transforms. Due to improve localization speed and localization accuracy, taking the advantage of the grey information, we decrease the number of edge points and parameter range down to a small range to locate iris boundary.

\subsection{A Recognition Perspective -}

For the choices of the classifier, we propose the use of a Gaussian Mixture Modeling (GMM) to model the posterior probability distribution of both iris texture and occlusion regions. GMMs have been widely used in all kinds of pattern recognition problems, including speech processing [21], human skin detection [22], realtime tracking [23], hazardous chemical agents detection [24], and bearing damage detection for induction motors [25]. The advantage of GMM is its modeling ability. As long as the number of Gaussians is large enough, GMM can model virtually any distribution. Another advantage of GMM is that its mathematical equation is easy to evaluate; thus the classification speed is very high during the test stage. This is an important consideration when dealing with problems like automatic mask generation because we have to perform pixelwise classifications for every iris image. The training of GMM uses Expectation-Maximization (EM) to optimize the parameters for Gaussian mixtures. EM-based parameter estimation for GMM training has a few drawbacks. First, the number of Gaussian mixtures has to be manually chosen. Second, the initialization of the mean of each Gaussian mixture is crucial. Similarly to the Kmeans algorithm, if the initial position of the mean of each Gaussian is not initialized appropriately, the EM algorithm may not be able to converge to the appropriate location of the mean of each Gaussian. Due to these two major drawbacks in the EM training algorithm, we decided to use an alternative training approach. Figueiredo and Jain proposed an unsupervised learning method for training GMMs [26]. This method can estimate the number of Gaussian mixtures without human intervention, and can avoid the boundary of the parameter space during the converging stage. The basic idea of Figueiredo-Jain's extension for GMM training (FJ-GMM algorithm) is that it dynamically adjusts the number of Gaussians by eliminating Gaussians which are not supported by the observation. The details of FJGMM training can be found in [26]. 


\section{Iris Normalization -}

Irises from different people may be captured in different size, and even for the iris from the same person, the size may change because of the variation of the illumination and other factors. Such elastic deformations in iris texture affect the results of iris matching. For the purpose of achieving more accurate recognition results, it is necessary to compensate for these deformations. Here, we anti-clockwise map the iris ring to a rectangular block of texture of a fixed size $(64 \times 512)$. According to the requirement of feature extraction, this block is then divided into eight smaller subimages. The size of each subimage is $64 \times 64$. An example of iris localization is shown in Figure 5aThe result after iris normalization is shown in Figure 5b.

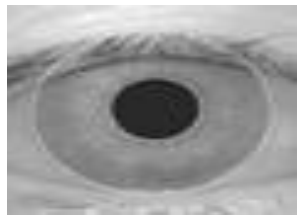

a)

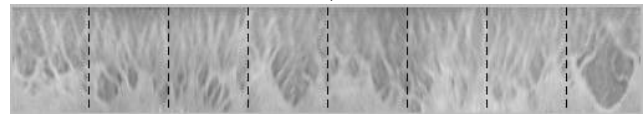

b)

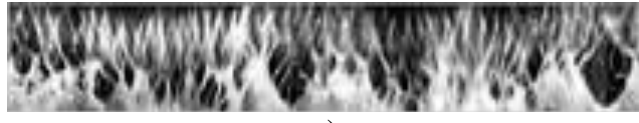

c)

Fig. 5 (a) Image after iris localization; (b) Eight unwrapped texture images; (c) Eight unwrapped texture images after enhancement and denoising.

The normalized iris image still has low contrast and may have non-uniform illumination caused by the position of light sources. All these may affect subsequent feature extraction and pattern matching. We enhance the iris image by means of local histogram equalization and remove high-frequency noise by filtering the image with a low-pass Gaussian filter. In order to obtain more well-distributed texture images, the 8 subimages are separately processed. Figure $2 \mathrm{~d}$ shows the preprocessing result of an iris image. From Fig. $5 \mathrm{~b}$ and $5 \mathrm{c}$, we can see that the method of enhancement and denoising is very effective.

\section{Feature set Extraction -}

After having obtained normalized arc segments for Iris texture areas, we execute Iris feature extraction for later comparison and verification tasks. Basically, our Iris feature extraction procedure consists of Gabor filters. The Gabor filter is the best to extract the salient features for the goal of classification between iris texture and occlusion artifacts. However, we had to decide which Gabor filter to use, i.e., what modulation frequency, orientation, and scale. In this section, we detail how we optimize the Gabor filter banks so the accuracy of the iris masks can be further enhanced. To achieve this goal, we employed an iterative nonlinear optimization approach. The mathematical equation of the generic Gabor filter is given below:

$$
\mathrm{z}(\mathrm{s}, \mathrm{t} ; \lambda, \theta, \psi, \sigma, \mathrm{Y})=\mathrm{e}-\left(\mathrm{s}^{\prime} 2+\mathrm{Y}^{\prime} 2 \mathrm{t}^{\prime} 2\right) / 2 \sigma^{\prime} 2 \cos \left(2^{\prime} \pi \mathrm{s}^{\prime} / \lambda+\psi\right),(1)
$$

where $S^{\prime}=\operatorname{scos} \theta+\operatorname{tsin} \theta$ and $\mathrm{t}^{\prime}=\sin \theta+\mathrm{t} \cos \theta$.

In this equation, $\lambda$ represents the wavelength of the wavelet, $\theta$ is the in-plane rotational angle of the filter, $\psi$ is the phase offset between the peak and the valley of the wavelet, $Y$ is the aspect ratio between the horizontal and vertical direction before the in-plane rotation, and it specifies the ellipticity of the support of the Gabor function; $\sigma$ specifies the variance of the global envelope (which corresponds to the frequency bandwidth of the band pass in the frequency domain

The Gabor filters have five parameters that need to be optimally selected to achieve the best discrimination for the goal of generating most accurate iris masks. However, because the final goal of automatic iris mask estimation is to generate an accurate mask in order to "improve iris recognition rate," we choose to use iris recognition performance as our objective function that we want to maximize. we are using this formula:

$$
\begin{aligned}
& \dot{z}=\arg \min \operatorname{FRR}(z(s, t ; \lambda, \theta, \psi, \sigma, Y)), \\
& { }^{\{} \lambda, \theta, \psi, \sigma, Y^{\}}
\end{aligned}
$$


where FRR0:1 stands for the False Reject Rate (FRR) when the False Accept Rate (FAR) equals 0.1 percent. The step to compute $\wedge^{\wedge} \mathrm{z}$ is as follows:

1. Given a specific set of Gabor parameter $(\mathrm{s}, \mathrm{t} ; \lambda, \theta, \psi, \sigma, \mathrm{Y})$ use the corresponding Gabor filter to extract a feature from the iris images of training set.

2. Use the feature set (consisting of pixel location, pixel intensity, and response from Gabor) to train GMM, with FJ-GMM training algorithm.

3. Estimate the iris occlusion map for every image in the test set.

4. Perform large-scale iris recognition using iris images and estimated occlusion map in the test set, then compute FRR at FAR $=0.1$ percent.

5. Use the result in step 4 as the output of cost function, then go back to step 1 to select a new Gabor parameter. Continue such loop until the best parameter ${ }^{\wedge} \mathrm{z}$ is found.

In step 4, when performing large-scale iris recognition, we basically follow Daugman's framework [3], [4], except that we used Haar wavelet for feature extraction. We choose to use Simulated Annealing (SA) method as our optimization algorithm. SA is a stochastic global optimization method which can

1.process cost functions which have an arbitrary degree of nonlinearities and discontinuities;

2. process arbitrary boundary conditions and constraints imposed on those cost functions;

3. be implemented quite easily with minimal effort of coding compared to other nonlinear optimization methods;

4. statistically guarantee the finding of an optimal solution [27].

\section{Conclusions -}

Accurate Iris Segmentation is fundamental for the success and precision of the sub-sequent feature extraction and recognition and consequently the high performance level of the Iris Recognition system. So, for accurate segmentation Iris Mask of high accuracy is needed to be derived during segmentation process. Iris boundaries are recognized by simple methods and less complex and faster algorithms than precious algorithms and it eliminates pupilary noises and reflections. This proposed techniques used guarantees the real-time Iris Segmentation even for Iris Images with severe occlusions. Therefore, high accuracy in Iris Segmentation leads to low error rate and high performance rate in Iris Recognition. The proposed segmentation approach is simple but extremely robust to various inputs of Iris images.

\section{References}

[1] J. Daugman, "High Confidence Visual Recognition of Persons by a Test of Statistical Independence," IEEE Trans. Pattern Analysis and Machine Intelligence, vol. 15, no. 11, pp. 1148-1161, Nov. 1993.

[2] J. Daugman, "Statistical Richness of Visual Phase Information: Update on Recognizing Persons by Iris Patterns," Int'1 J. Computer Vision, vol. 45, no. 1, pp. 25-38, 2001.

[3] J. Daugman, "How Iris Recognition Works," Proc. Int'l Conf. Image Processing, vol. 1, pp. I-33-I-36, 2002.

[4] J. Daugman, "Biometric Personal Identification System Based on Iris Analysis," US Patent 291560, 1994.

[5] J. Daugman, "How Iris Recognition Works," IEEE Trans. Circuits and Systems for Video Technology, vol. 14, no. 1, pp. 21-30, Jan. 2004.

[6] L. Ma, T. Tan, Y. Wang, and D. Zhang, "Personal Identification Based on Iris Texture Analysis," IEEE Trans. Pattern Analysis and Machine Intelligence, vol. 25, no. 12, pp. 1519-1533, Dec. 2003.

[7] C. Tisse, L. Martin, L. Torres, and M. Robert, "Person Identification Technique Using Human Iris Recognition," citeseer.ist.psu. edu/tisse02person.html, 2002.

[8] J. Daugman, "New Methods in Iris Recognition," IEEE Trans. Systems, Man, and Cybernetics, Part B, vol. 37, no. 5, pp. 1167-1175, Oct. 2007.

[9] W. Kong and D. Zhang, "Accurate Iris Segmentation Based on Novel Reflection and Eyelash Detection Model,” Proc. Int'l Symp. Intelligent Multimedia, Video, and Speech Processing, pp. 263-266, 2001.

[10] J. Zuo, N. Kalka, and N. Schmid, "A Robust Iris Segmentation Procedure for Unconstrained Subject Presentation," Proc. Special Session on Research at the Biometric Consortium Conf. Biometrics Symp., pp. 1-6, Aug. 21-Sept. 19, 2006.

[11] E. Krichen, S. Garcia-Salicetti, and B. Dorizzi, "A New Probabilistic Iris Quality Measure for Comprehensive Noise Detection," Proc. First IEEE Int'l Conf. Biometrics: Theory, Applications, and Systems , pp. 1-6, Sept. 2007.

[12] J. Thornton, "Matching Deformed and Occluded Iris Patterns: A Probabilistic Model Based on Discriminative Cues," PhD thesis, Dept of Electrical and Computer Eng., Carnegie Mellon Univ., 2007. 
[13] Z. Sun, G. Bebis, and R. Miller, “On-Road Vehicle Detection Using Evolutionary Gabor Filter Optimization,” IEEE Trans. Intelligent Transportation Systems, vol. 6, no. 2, pp. 125-137, June 2005.

[14] Z. Sun, G. Bebis, and R. Miller, "Evolutionary Gabor Filter Optimization with Application to Vehicle Detection," Proc. Third IEEE Int'l Conf. Data Mining, pp. 307-314, Nov. 2003.

[15] M. Afshang, M. Helfroush, and A. Zahernia, "Gabor Filter Parameters Optimization for Texture Classification Based on Genetic Algorithm," Proc. Second Int'l Conf. Machine Vision, pp. 199-203, Dec. 2009.

[16] M. Li and R. Staunton, "Unsupervised Texture Segmentation Based on Immune Genetic Algorithms and Fuzzy Clustering,” Proc. Eighth Int'l Conf. Signal Processing, vol. 2, Nov. 2006.

[17] J. Zhou, Z. Ji, L. Shen, Z. Zhu, and S. Chen, "PSO Based Memetic Algorithm for Face Recognition Gabor Filters Selection,” Proc. IEEE Workshop Memetic Computing, pp. 1-6, Apr. 2011.

[18] Y.-N. Zhao, Z. Jin, J.-Y. Yang, C.-C. Liu, and X.-Q. Meng, "Practical Parameers Design for Gabor Filers with Application to Face Recognition," Proc. Int'l Conf. Machine Learning and Cybernetics, vol. 4, pp. 2229-2234, Aug. 2007.

[19] C. Tsai, J. Taur, and C. Tao, "Iris Recognition Using Gabor Filters Optimized by the Particle Swarm Technique," Proc. IEEE Int'l Conf. Systems, Man, and Cybernetics, pp. 921-926, Oct. 2008.

[20] J. Thornton, M. Savvides, and B. Kumar, "An Evaluation of Iris Pattern Representations," Proc. First IEEE Int'l Conf. Biometrics: Theory, Applications, and System, pp. 1-6, Sept. 2007.

[21] L. Rabiner and B.-H. Juang, Fundamentals of Speech Recognition. Prentice-Hall, Inc., 1993.

[22] M.-H. Yang and N. Ahuja, "Gaussian Mixture Model for Human Skin Color and Its Applications in Image and Video Databases," Storage and Retrieval for Image and Video Databases VII, vol. 3656, no. 1, pp. 458-466, http://link.aip.org/link/?PSI/3656/458/1, 1998.

[23] C. Stauffer and W. Grimson, "Adaptive Background Mixture Models for Real-Time Tracking," Proc. IEEE Conf. Computer Vision and Pattern Recognition, vol. 2, p. 2246, 1999.

[24] J. Ilonen, J.-K. Kamarainen, H. Kalviainen, and O. Anttalainen, "Automatic Detection and Recognition of Hazardous Chemical Agents,” Proc. 14th Int'l Conf. Digital Signal Processing, vol. 2, pp. 1345-1348, 2002.

[25] T. Lindh, J. Ahola, J. Kamarainen, V. Kyrki, and J. Partanen, "Bearing Damage Detection Based on Statistical Discrimination of Stator Current," Proc. Fourth IEEE Int'l Symp. Diagnostics for Electric Machines, Power Electronics, and Drives, pp. 177-181, Aug.2003.

[26] M. Figueiredo and A. Jain, "Unsupervised Learning of Finite Mixture Models," IEEE Trans. Pattern Analysis and Machine Intelligence, vol. 24, no. 3, pp. 381-396, Mar. 2002.

[27] D. Bertsimas and J. Tsitsiklis, "Simulated Annealing," Statistical Science, vol. 8, no. 1, pp. 10-15, 1993.

[28] J. M. Ali and A. E. Hassanien, An Iris Recognition System to enhance E-Security, Advanced Modeling and Optimization, vol. 5, pp. 93-104. 